\title{
Antenna Pointing for High Bandwidth Communications from Mobile Robots
}

\author{
Deepak Bapna, Eric Rollins, Alex Foessel, Red Whittaker \\ Email:deepak@ri.cmu.edu
}

\author{
Field Robotics Center, The Robotics Institute \\ Carnegie Mellon University \\ Pittsburgh PA 15213
}

\begin{abstract}
This paper discusses the challenge of achieving high bandwidth, distant range wireless communication from mobile robots by way of antenna tracking. In the case of robots traversing rough terrain at moderate speeds, tracking demands high slew rates and large motion ranges due to vehicle motion disturbances. Attaining tracking accuracy, particular with the low mass and power inherent to mobile robots, requires an innovative approach. This paper presents requirements analysis, mechanism design, sensor configuration and some experimental results for an antenna pointing mechanism that was developed for Nomad, a planetary-relevant mobile robot. The mechanism was demonstrated during the summer of 1997 in Nomad's $200 \mathrm{~km}$ traverse in the Atacama Desert of Chile.
\end{abstract}

\section{Introduction}

The agenda for robotic exploration of planetary surfaces is growing. Proposed missions have ambitious goals in terms of exploration, information transmission and low cost. For example, there is a near term robotic mission planned for Antarctica to search for meteorites [6]. Another proposed mission is to search for ice at the lunar pole [5]. Such missions require high data rate transmission from the robot to a distant control station at relatively low power levels (as total power is limited), possible only if the angular offset between transmitter and receiver antennas is small ([1], [3]). By maintaining line-of-sight lock between the transmitting antenna on a robot and the receiving antenna on the control station, a precision tracking capability can enable the bandwidth required for such distant range robotic exploration. This ability to achieve tracking while roving is important for a wide variety of mobile robot tasks including teleoperation, optical communication, mobile surveying and reconnaissance, cooperative manipulation, and active vision.
Mobile payload tracking, generally requires high slew rates and large articulation ranges in order to stabilize and aim a payload while moving over uneven terrain. Traditional approaches typically append a pan/tilt (or gimbal) to the moving vehicle or platform. Although precision can be on the order of $\mu \mathrm{rad}$, these pointing mechanisms are massive and require high power. Antenna dishes on deep sea drilling platforms are for stance, stabilized and aimed during rough seas with massive high power positioners. Likewise, a gun barrel aiming system on an M-1 tank tracks targets while moving at high speed but is quite massive. Mobile robots, however, are usually limited in mass, power and available space, making the payload tracking particularly challenging.

This work is concentrated on wheeled robots moving at moderate speeds in rough terrains. The specific application considered is transmission of high resolution imagery from a robot moving in natural terrain to a distant control station.

\section{Atacama Desert Trek}

In summer 1997, Carnegie Mellon University conducted the Atacama Desert Trek, a demonstration of robotic technologies necessary for planetary exploration [1]. In this demonstration the robot Nomad (Figure 1),

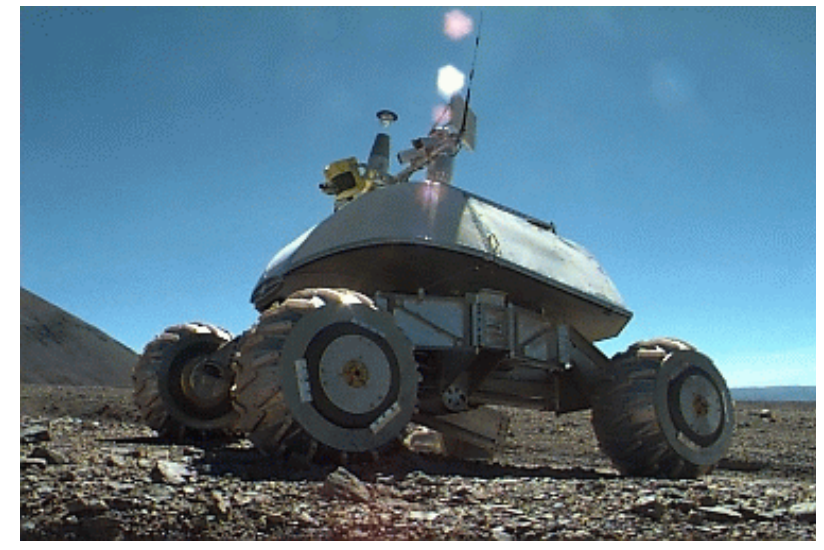

Figure 1: Nomad 
operated in hands-off mode for two months and traversed $220 \mathrm{~km}$ in the planetary-like Atacama Desert of Chile. Nomad was controlled from remote stations located at the Carnegie Science Center in Pittsburgh, PA, NASA Ames in Mountain View, CA and Entel Headquarters in Santiago, Chile. The trek demonstrated such key technologies as planetary locomotion, panospheric imaging for effective teleoperation, autonomous navigation, safeguarded teleoperation, and, notably high bandwidth communication. In addition to advancing robotics technologies for planetary exploration, the Desert Trek involved mass public participation in robotic exploration for the first time. Nomad's rich, interactive user interface and safeguarded teleoperation presented novice operators with the opportunity to operate Nomad safely from the distant control centers. Images and data from Nomad were also immediately available on the Internet.

The principal tool for enabling public participation was a high resolution panospheric camera [8]. In order to transport its imagery, generated at 4-6 Hz, from the rover to a local control station and then to remote mission control sites, a high bandwidth (1.4 Mbps) reliable communication link was paramount. The ideal scenario was to transmit from the rover directly to a satellite and from there to control stations [3]; however, the required size and mass of the antenna dish and transmitter equipment made this scenario infeasible for this category of robots. The next option was to transmit to a nearby relay station (located within 8-10 $\mathrm{km}$ ) which then would retransmit the information to a satellite (Figure 2). Because these

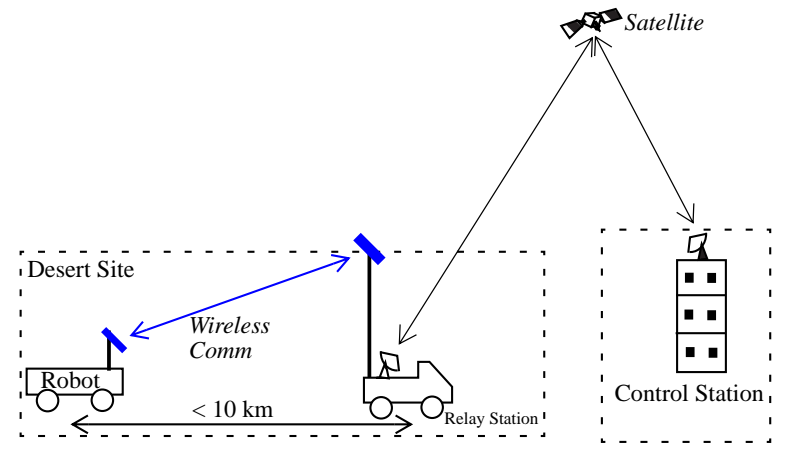

Figure 2: Communication Scenario

requirements (1.4 Mbps, $10 \mathrm{~km}$ range) could not be achieved using omnidirectional antennas, high gain directional antennas were required. Further, the directional antenna on the rover needed to be pointed toward the receiving antenna on the relay station to maintain the communication link while the robot was moving.

The pointing requirements, described in the next section, were met only by a custom designed system. The remainder of this paper describes the antenna pointing mechanism design, sensing scenarios, and some experimental results.

\section{Requirements}

The pointing requirements are presented in Table 1.

\begin{tabular}{|c|c|}
\hline Item & Value \\
\hline \hline Elevation range & $-40^{\circ}$ to $+60^{\circ}$ \\
\hline Azimuth range & continuous \\
\hline Elevation Rate & $60^{\circ} / \mathrm{s}$ \\
\hline Azimuth Rate & $30^{\circ} / \mathrm{s}$ \\
\hline Elevation Acceleration & $190^{\circ} / \mathrm{s}^{2}$ \\
\hline Azimuth Acceleration & $190^{\circ} / \mathrm{s}^{2}$ \\
\hline Pointing Accuracy & $2.5^{\circ}$ \\
\hline Pay Load Mass & $2 \mathrm{~kg}$ \\
\hline Payload Size & $27.3 \times 34 \times 2 \mathrm{~cm}$ \\
\hline Target Mass & $\sim 10 \mathrm{~kg}$ \\
\hline Target Power & $\sim 25 \mathrm{~W}$ \\
\hline
\end{tabular}

Table 1: Antenna Pointing Requirements

The range is a combination of three factors:

- Motion of the rover, e.g., rocking and turning

- Terrain characteristics e.g., slopes and steps

- Distance from the relay station

The maximum slope the rover could traverse was $\pm 35^{\circ}$. The maximum obstacle size the rover could climb

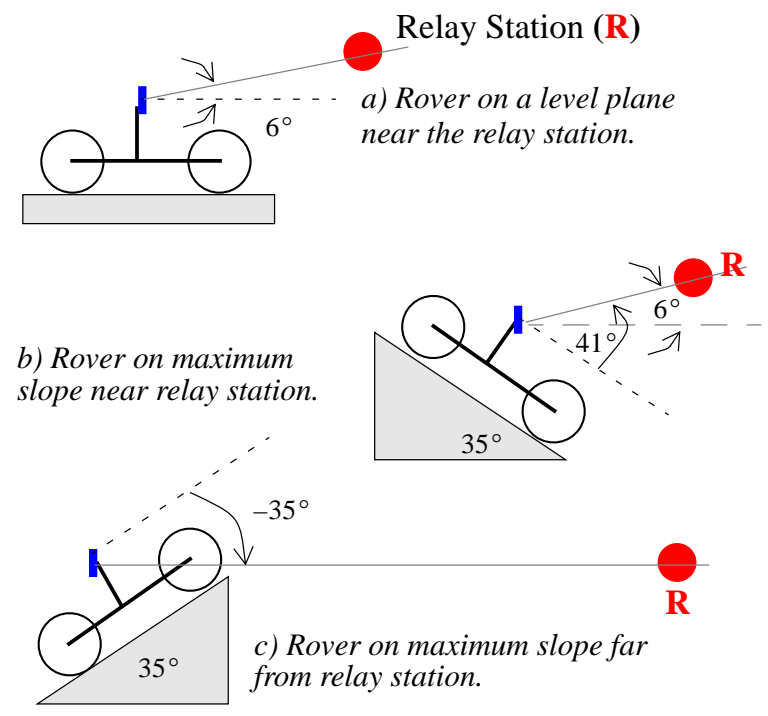

Figure 3: Pointing Range

from level terrain was $50 \mathrm{~cm}$, which pitched the rover by $19^{\circ}$. The rover was subjected to tipover at $\pm 35^{\circ}$ pitch. 
Therefore, the pointing excursions from the first two factors were $\pm 35^{\circ}$. The placement of the receiver antenna on a hill put the height at approximately $100 \mathrm{~m}$. At maximum range, when the rover was 8-10 km away from the receiver antenna, the elevation angle of the antenna with the rover on flat ground was effectively zero. Restricting the minimum range to $1 \mathrm{~km}$, the corresponding angle was approximately $6^{\circ}$. As shown in Figure 3, the total elevation required was $-35^{\circ}$ to $+41^{\circ}$. Including a safety factor, the antenna pointing mechanism was specified for $-40^{\circ}$ to $+60^{\circ}$. The azimuth specified was continuous.

The elevation rate was approximated as the rate at which the angle changes when the robot falls from a $50 \mathrm{~cm}$ step (Figure 4) The rates and accelerations shown in Table 1 are based on a trapezoidal velocity profile and include a safety factor.

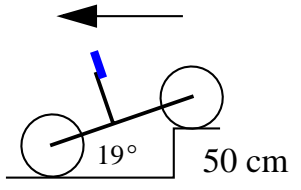

$$
\begin{aligned}
\dot{\theta} & =\frac{\theta-\theta_{0}}{t} \\
& =\frac{19-0}{0.64} \\
& =29.60^{\circ} / \mathrm{s}
\end{aligned}
$$

$$
\mathrm{t}=0.64 \mathrm{~s} \text {, time to freefall } 50 \mathrm{~cm}
$$

Figure 4: Pointing Rates

Pointing accuracy of $\pm 2.5^{\circ}$ was based on the radiation patterns of the antenna.

The following table gives specifications of some other antenna pointing systems for comparison. Toshiba APM-1

\begin{tabular}{|c|c|c|c|c|}
\hline & $\begin{array}{c}\text { Toshiba } \\
\text { APM-1 }\end{array}$ & $\begin{array}{c}\text { HST } \\
\text { STAPS }\end{array}$ & $\begin{array}{c}\text { KVH } \\
\text { TracVision }\end{array}$ & $\begin{array}{c}\text { CMU } \\
\text { (objective })\end{array}$ \\
\hline \hline Excursion & $\begin{array}{c}+/-15 \\
\text { both axes }\end{array}$ & $\begin{array}{c}+/-110 \mathrm{x} \\
+/-110 \mathrm{y}\end{array}$ & $\begin{array}{c}+/-110 \mathrm{El} \\
\text { Az. Cont. }\end{array}$ & $\begin{array}{c}+/-60 \mathrm{El} \\
\text { Az. Cont. }\end{array}$ \\
\hline Rate $\left({ }^{\circ} / \boldsymbol{s}\right)$ & 0.31 & 0.5 & 12 & 60 \\
\hline Mass $(\mathbf{k g})$ & 9.90 & 17.2 & 10.1 & 10 \\
\hline Power $(\mathbf{W})$ & $\begin{array}{c}39 \text { Peak, } \\
4.5 \mathrm{Avg}\end{array}$ & $30 \mathrm{Nom}$ & $\begin{array}{c}54 \text { Peak } \\
28 \text { Nom }\end{array}$ & 25 \\
\hline Accuracy $\left({ }^{\circ}\right)$ & 0.0029 & 0.6 & 3 & 2.5 \\
\hline
\end{tabular}

Table 2: Comparison of various mechanisms

[4] and HST STAPS [4] are antenna positioners for satellite applications while KVH's TracVision [7] is for antenna pointing from boats. As seen from the table, the pointing rates for these applications are much lower than the rates required for pointing from mobile robots and therefore are not useful for the purpose.

\section{Configuration}

Several configurations for the antenna pointing mechanism were considered. In particular, two-stage pointing, direct drive gimbal, and gyroscopic stabilization were assessed in light of such factors as mass, power, size, cost, manufacturability, ability to use off-the-shelf components, reliability, coupling, and controllability. A two stage pointing device could isolate gross vehicle rotations using one mechanism (active like gyroscopic stabilization or passive like pendulum) and perform finer rotations with another mechanism (for example, a gimbal). While providing better performance, two-stage pointing was deemed as "overkill" for this application due to low accuracy requirements. Extensively used in satellite applications, gyroscopic stabilization provides better isolation of vehicle motion, but precession induced by high disturbance torques (due to vehicle motion and motion of the RF cable) would be problematic in mobile robot applications.

The final configuration chosen and developed was a single stage, pan/tilt mechanism. Each motion incorporated a brushed motor and harmonic drive reduction. Figure 5 shows the configuration. The main features of the

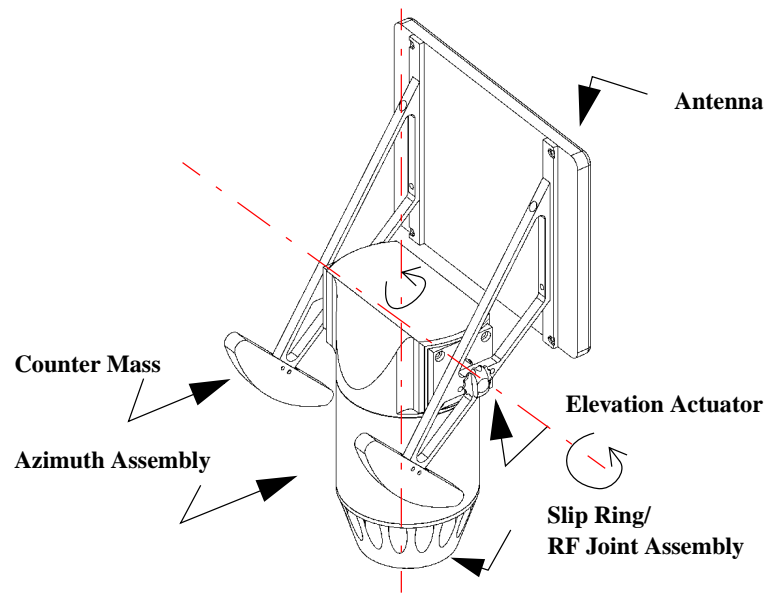

Figure 5: Antenna Pointing Mechanism

mechanism are:

1. Azimuth/Elevation configuration: The mechanism consists of an azimuth and an elevation assembly. The elevation actuator lies above the azimuth actuator. This configuration has less coupling compared to $\mathrm{x} / \mathrm{y}$ configuration (e.g., used for HST STAPS [4]).

2. Slip ring for elevation actuator: A slip ring is used for power, sensing (encoder signal) and command of the elevation actuator. Slip rings, although considered more complex and less reliable than cable-wraps, are suitable for this application for several reasons. A slip ring: 
- allows continuous rotation, unlike cable-wrap.

- reduces complexity as management of cables to avoid minimum bend radii (of RF flexible cable and other wires) is difficult in a compact package.

- does not introduce unpredictable torques which are inherent in a cable-wrap solution.

3. $R F$ rotary joint: An RF rotary joint is used to send $\mathrm{RF}$ signals from the transmitter to the antenna. The RF rotary joint is co-axial with the slip ring. The RF rotary joint has certain advantages over flexible cable:

- The RF characteristics remain nearly constant. With flexible cable, the RF characteristics change randomly with motion of the cable.

- A RF rotary joint introduces less losses. Typical loss in flexible RF cable is about $0.2 \mathrm{~dB} / \mathrm{ft}$. Total loss using cable-wrap is about $1.2 \mathrm{~dB}$ compared to $0.5 \mathrm{~dB}$ for RF rotary joint.

4. Harmonic drive and brushed motor: Brushed motors are used with harmonic reduction for both azimuth and elevation actuators. The primary rationale was to minimize backlash. A typical off-the-shelf planetary train has a backlash about $1^{\circ}-2^{\circ}$, unsuitable for the application.

5. Balanced system: The payload is balanced using a counter mass. This minimizes disturbances torques, thus reducing the size of the actuators and complexity of the controller.

This configuration using RF rotary joint, slip rings and balanced payload proved very efficient for this application.

\section{Sensors}

Several sensors are required to interpret the position of the receiver antenna, robot position, robot orientation and azimuth and elevation angles. The following sensors were used for antenna pointing:

DGPS: A Differential Global Positioning System was used to derive the position of the robot and the relay station in the inertial frame. One GPS unit was mounted on the rover, another (the master unit) at the relay station. The two receivers were used in a differential configuration to provide good position accuracy.

Compass: A stabilized gyro compass was used for magnetic azimuth of the vehicle.

Inclinometers: Inclinometers delivered the roll and pitch of the vehicle. The azimuth digital gyro compass (ADGC) specified gave roll and pitch in addition to the magnetic azimuth.

Encoders: Incremental encoders mounted on the motors were used to sense the positions of azimuth and elevation motors. Optical switches were used as limits on the output end of azimuth and elevation and to generate absolute signal.

The block diagram of the controller is shown in Figure 6 . The kinematic module computed the required positions of the azimuth and elevation motors $\left(X_{\text {ref }}\right)$ using data from various sensors. The error, $e$, was computed by subtracting the actual position of the motors $(X)$. Controller was a PID control which commanded amplifiers to drive the two motors.

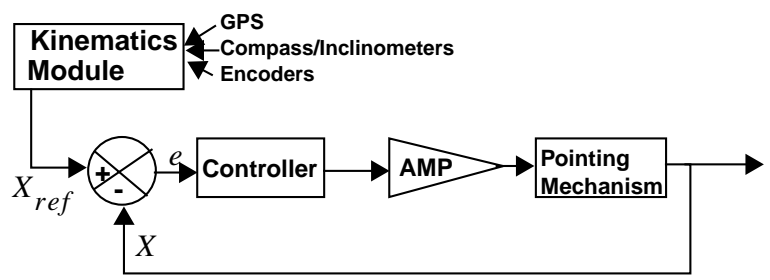

Figure 6: Block diagram for Pointing

As evidenced from the block diagram, there is no means to measure the actual pointing angle (in global reference frame). The output was the position of each motor $(X)$. Error was introduced due to several factors including the accuracy, resolution and bandwidth of each sensor, backlash in the drive train, and time constant of the system. There was no feedback from the receiver antenna and hence it was an "open loop" control strategy. It was, however, sufficient for this application as detailed in the following section.

\section{Kinematics and Error Analysis}

It is important to verify the sufficiency of sensor accuracies. Pointing error due to each sensor was estimated by differentiating the inverse kinematics equations.

As shown in Figure 7, the following reference frames were defined for the purpose of analysis and control:

\section{$\{G\}$ Global reference frame}

$\{$ V\} Vehicle reference frame

$\{A\}$ Azimuth reference frame

$\{$ E $\}$ Elevation reference frame

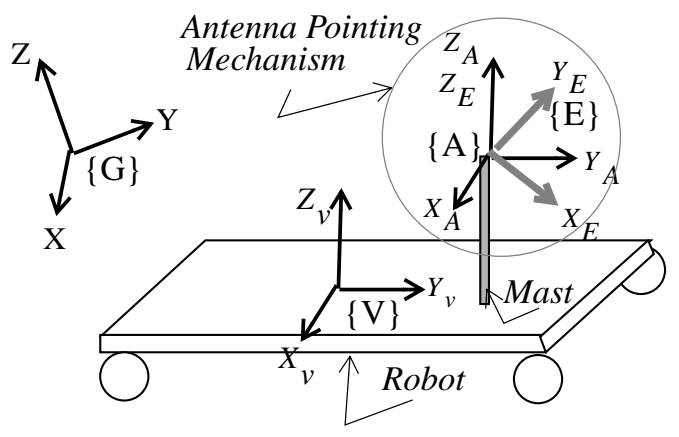

Figure 7: Reference Frames 
The global reference frame was fixed w.r.t. the Earth and its X-axis pointed east, Y-axis pointed North and Zaxis pointed upwards. As we were interested in the relative position of the rover and the relay station, the origin of the frame was of minor importance. The Y-axis of the vehicle reference frame pointed forward, the $\mathrm{X}$-axis pointed to the right side of the vehicle, and the Z-axis pointed up. The vehicle frame was located at the compass center. Azimuth $\{A\}$ and Elevation $\{E\}$ were the two frames associated with the antenna. The origin of these two frame was located at the intersection of azimuth and elevation axis. The Z-axis of the azimuth frame was normal to the vehicle pointing upwards while the y-axis pointed in the direction of vehicle travel. The elevation frame was obtained by rotating the azimuth frame around azimuth $\mathrm{Z}$-axis by $\gamma_{p}$, the azimuth angle.

The standard z-y-x Euler angle representation was used for analysis. The required azimuth and elevation angles for line-of-sight pointing were given by:

$\alpha_{p}=\operatorname{atan}(-X / Y)$ and $\gamma_{p}=\operatorname{atan}\left(Z /\left(\sqrt{X^{2}+Y^{2}}\right)\right)$

where $X, Y, Z$ are defined as

$$
\begin{gathered}
X=-x_{p}+\left(x_{r}-x_{v}\right) \cos \alpha \cos \beta+\left(y_{r}-y_{v}\right) \sin \alpha \cos \beta \\
-\left(z_{r}-z_{v}\right) \sin \beta \\
Y=-y_{p}+\left(x_{r}-x_{v}\right)\{\cos \alpha \sin \beta \sin \gamma-\sin \alpha \cos \gamma\} \\
+\left(y_{r}-y_{v}\right)\{\cos \alpha \cos \gamma+\sin \alpha \sin \beta \sin \gamma\} \\
+\left(z_{r}-z_{v}\right) \cos \beta \sin \gamma \\
Z=-z_{p}+\left(x_{r}-x_{v}\right)\{\cos \alpha \sin \beta \cos \gamma+\sin \alpha \sin \gamma\} \\
+\left(y_{r}-y_{v}\right)\{\sin \alpha \sin \beta \cos \gamma-\cos \alpha \sin \gamma\} \\
+\left(z_{r}-z_{v}\right) \cos \beta \cos \gamma
\end{gathered}
$$

Here,

$$
\alpha_{p}
$$

$\gamma_{p}$

$\left(x_{r}, y_{r}, z_{r}\right)$

$\left(x_{v}, y_{v}, z_{v}\right)$

$\left(x_{p}, y_{p}, z_{p}\right)$

Required Azimuth Angle (Encoder)

Required Elevation Angle (Encoder)

Coordinates of Relay Station (GPS)

Coordinates of the Vehicle (GPS)

Coordinates of the pointing mechanism in the vehicle reference frame

$(\alpha, \beta, \gamma) \quad$ (yaw, roll, pitch) of the vehicle (ADGC)

The error analysis was performed by differentiating the expressions for $\alpha_{p}$ and $\gamma_{p}$ as:

$$
d \alpha_{p}=\sum_{i}\left|\frac{\partial \alpha_{p}}{\partial x_{i}} d x_{i}\right| \quad \text { and } \quad d \gamma_{p}=\sum_{i}\left|\frac{\partial \gamma_{p}}{\partial x_{i}} d x_{i}\right|
$$

where $x=\left[x_{r}, y_{r}, z_{r}, x_{v}, y_{v}, z_{v}, \alpha, \beta, \gamma, \alpha_{p}, \gamma_{p}\right]$, is the vector of generalized variables.
The analysis was performed for the following two configurations:

$$
\begin{aligned}
& x 1=\left[0,0,100,1000,1000,0, \pi, \frac{\pi}{6}, \frac{\pi}{6}, \alpha_{p}, \gamma_{p}\right], \text { and } \\
& x 2=\left[0,0,100,1000,1000,0, \pi, 0,0, \alpha_{p}, \gamma_{p}\right]
\end{aligned}
$$

Table 3 presents the results of the analysis. The second column cites the expected accuracies of each sensor listed in the first column. The third and forth columns give the error in elevation $\left(\gamma_{p}\right)$ and azimuth $\left(\alpha_{p}\right)$, respectively, due to each sensor; this is calculated by assuming the error due to other sensors is zero. The last column lists the criticality (C) of the sensor to achieve pointing accuracy. As seen from the table, Compass and Inclinometers are the most critical sensors for accuracy while GPS is the least critical.

The expected cumulative error due to sensor

\begin{tabular}{|c|c|c|c|c|}
\hline Sensor & $\begin{array}{c}\text { Sensor } \\
\text { Accuracy }\end{array}$ & $\begin{array}{c}\text { Elevation } \\
\text { Error }\end{array}$ & $\begin{array}{c}\text { Azimuth } \\
\left.\text { Error }{ }^{(}\right)\end{array}$ & $C^{i}$ \\
\hline \hline \multicolumn{5}{|c|}{ Configuration 1 } \\
\hline GPS & $20 \mathrm{~cm}$ & 0.045 & 0.039 & $\mathrm{~L}$ \\
\hline Compass & $1.0^{\circ}$ & 0.650 & 0.750 & $\mathrm{H}$ \\
\hline Inclinometers & $0.5^{\circ}$ & 0.658 & 0.2515 & $\mathrm{H}$ \\
\hline Az. Encoder & $3.6^{\circ}$ & 0.0 & 0.024 & $\mathrm{~L}$ \\
\hline Elev. Encoder & $3.6^{\circ}$ & 0.041 & 0.0 & $\mathrm{~L}$ \\
\hline Total & - & 1.394 & 1.065 & - \\
\hline \multicolumn{5}{|c|}{ Configuration 2} \\
\hline GPS & $20 \mathrm{~cm}$ & 0.041 & 0.017 & $\mathrm{~L}$ \\
\hline Compass & $1.0^{\circ}$ & 0.0 & 1.002 & $\mathrm{H}$ \\
\hline Inclinometers & $0.5^{\circ}$ & 0.707 & 0.025 & $\mathrm{H}$ \\
\hline Az. Encoder & $3.6^{\circ}$ & 0.0 & 0.024 & $\mathrm{~L}$ \\
\hline Elev. Encoder & $3.6^{\circ}$ & 0.041 & 0.0 & $\mathrm{~L}$ \\
\hline Total & - & 0.789 & 1.068 & - \\
\hline
\end{tabular}

Table 3: Pointing Errors due to Sensor Inaccuracies

i. Criticality to achieve pointing accuracy. H- High, M-Medium, L- Low

inaccuracies was approximately $1.5^{\circ}$. This met the accuracy requirements of this application and hence open loop control was sufficient. When more accurate pointing is required, closed-loop pointing is necessary. In that case, there needs to be a feedback from the target about the actual pointing angle. This can be achieved by the measurement of signal power strength. 


\section{Experiments and Results}

The antenna pointing mechanism performed without failure during the two months of the Atacama Desert Trek. It enabled communication at ranges up to $11 \mathrm{~km}$, a record to our knowledge.

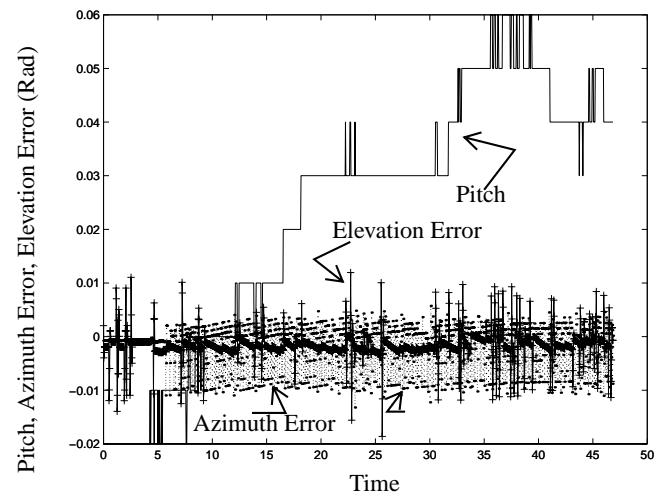

Figure 8: Pointing Error

During the trek, various tests were conducted to evaluate performance of the antenna pointing mechanism. Figure 8 depicts the results of one test in which the robot was commanded to traverse an obstacles field. As seen, the controller assures that the error is always zero.

Figure 9 shows the input data rate on the router recorded at random times for a period of about one hour. During this time, pointing experiments were performed as the robot was commanded to move in circles on difficult terrains (obstacle fields) at different velocities. As can be seen, even though the data rate fluctuated, it remained fairly high at all times. This graph offers a qualitative estimate of the pointing mechanism's performance.

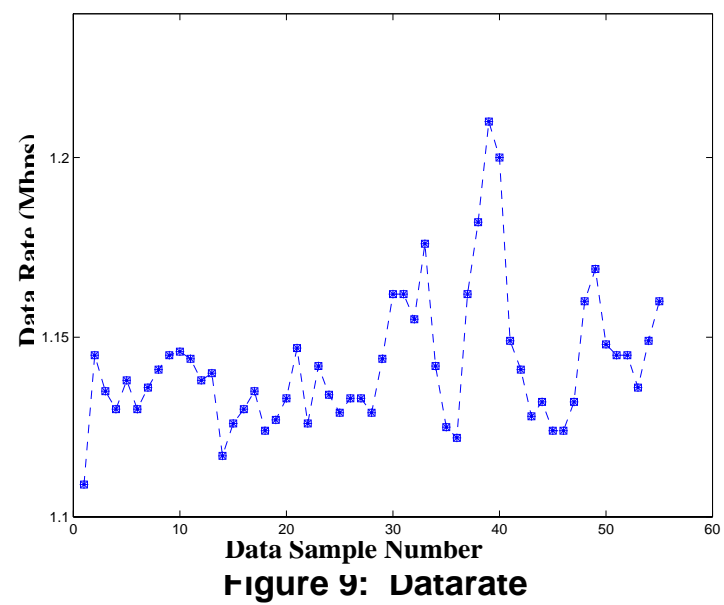

As clear from Figure 8, controller errors were of the order of $0.6^{\circ}$ (0.01 rad) maximum. As discussed in the previous section, the expected cumulative error due to sensors was approximately $1.6^{\circ}$ (estimated). Therefore, the estimated total pointing error was $\leq 2.2^{\circ}$ nominally, which is within the allowable range. Considering this and because images were continuously received at the control station, and a communication range of up to $11 \mathrm{~km}$ was achieved, it can be deduced that the link performed appropriately.

\section{Conclusions and Future Work}

This paper introduces active antenna pointing, a novel solution to the problem of high data rate, long range communication from outdoor mobile robots. The requirement analysis, mechanism design, sensor configuration and key results for the antenna pointing mechanism used in the Atacama Desert Trek are presented. The system was based on open loop and proved adequate for this application due to the large beamwidth of the antenna. It enabled communication to a record range of 11 $\mathrm{km}$. In future, closed-loop control would be incorporated and the mechanism would be used to establish direct communication with the satellite.

\section{References}

[1] Bapna, D., "Payload Pointing from Mobile Robots", Ph.D. dissertation, The Robotics Institute, Carnegie Mellon University, March 1998.

[2] Bapna, D., Teza, J.P., Rollins, E., and Whittaker, W., "An Innovative High Bandwidth Communication System for Mobile Robots", 27th International Conference on Environmental Systems, Lake Tahoe, Nevada, July 14-17, 1997, SAE Technical Series, Paper 972488.

[3] Bapna, D.; Martin, M.; and Whittaker, W. "Earth-Moon Communication from a Moving Lunar Rover", Proceedings of the 42nd International Instrumentation Symposium, San Diego, CA, May 5-9, 1996, pp613-622.

[4] Hyde, G., ed. "NASA/NSF Panel Report on- Satellite Communications Systems and Technology", International Technology Research Institute, JTEC/WTEC Program, Loyala College in Maryland, July, 1993.

[5] "Lunar Ice Discovery", Discovery Proposal, Carnegie Mellon University, Pittsburgh, PA, 1997.

[6] "Meteorite Search Technology", NASA TRIWG proposal, Carnegie mellon University, Pittsburgh, PA, 1997.

[7] TracVision, KVH Industries, Inc., Middleton, RI, USA, "http://www.kvh.com/tracvisi/tracvisi.html".

[8] Whittaker, R., Bapna, D., Maimone, M., and Rollins, E., "Atacama Desert Trek: A Planetary Analog Field Experiment", Proceedings of i-SAIRAS 97, Tokyo, Japan, July 14-16, 1997, pp355-360.

\section{Acknowledgments}

This research is funded by NASA CodeX (telerobotics group) through grants NAGW-3863 and NAGW-1175.

We are thankful to BEI and Trimble for sensor donations. We would also like to thank all the members of "Atacama Desert Trek" team at Field Robotics Center, CMU for their cooperation. 